\title{
UNA CASA PARA LA REVOLUCION: UN PROYECTO DE LEGITIMACIÓN CULTURAL EN CUBA (1959-1965)
}

GREYSER COTO SARDINA ${ }^{1} /$ GRETHEL DOMENECH HERNÁNDEZ ${ }^{2}$ Instituto de Historia de Cuba/ Universidad de las Artes -ISA-

Calzada Santiago, № 61 Rincón, Boyeros / Delicias, № 292 e, Quiroga y Colina. Lawton, 10 de octubre

Código Postal 2303, La Habana, Cuba/ Código Postal 2303, La Habana, Cuba.

\section{grey@ihc.cu/ domenech@isa.cult.cu}

\section{RESUMEN}

Con el triunfo revolucionario del 1ro de enero de 1959 en Cuba, se inicia una profunda transformación de todas las estructuras socioeconómicas, políticas y culturales. Como parte de esta dinámica comienza un proceso de institucionalización que en el ámbito de la cultura encuentra uno de sus mayores referentes en la creación de Casa de las Américas en julio de 1959. Las necesidades de legitimación del nuevo sistema hallaron en la institución un mecanismo para fomentar y estimular las ideas revolucionarias y su proyección hacia América Latina. En el período de 1959 a 1965, a través de sus principales acciones, dígase su revista y concurso, refrendaron el compromiso intelectual como fundamento principal de su discurso.

Palabras clave: Revolución Cubana, Casa de las Américas, institucionalización, compromiso intelectual, legitimación, cultura.

\footnotetext{
${ }^{1}$ Licenciada en Historia por la Universidad de la Habana. Investigadora en el Instituto de Historia de Cuba. Cuba.

${ }^{2}$ Licenciada en Historia por la Universidad de la Habana. Profesora Instructora del Departamento de Estudios Cubanos de la Universidad de las Artes (ISA). Cuba.
} 
A HOUSE FOR THE REVOLUTION: A PROJECT OF CULTURAL LEGITIMATION IN CUBA (1959-1965)

\section{ABSTRACT}

With the revolutionary triumph I Cuba, a deep transformation of all the socioeconomic, political and cultural structures is started. As part of this dynamics a process of institutionalization which finds in the scope of culture one of its higher referents in the creation of Casa de las Americas in July 1959 starts. The needs of legitimation of the new system found in the institution a mechanism to encourage and stimulate the revolutionary ideas and its projections towards Latin-America. In the period from 1959 to 1965 , through its main actions, understood as its magazine and contest, the intellectual compromise as principal foundation of its discourse was authenticated.

Key words: Cuban Revolution, Casa de las Américas, institutionalization, intellectual compromise, legitimation, culture.

\section{Introducción}

El triunfo de la Revolución Cubana, el 1ro de enero de 1959, significó una profunda transformación que marcó un antes y un después para Cuba y América Latina. Visto desde la política exterior de los Estados Unidos hasta el giro y la fortaleza de la izquierda latinoamericana, la historia de nuestro continente no puede obviar dicho cambio. Afirmaba Alfaro Sequeiros, que la Revolución Cubana era el movimiento político-social más trascendente de la historia contemporánea de todo el ámbito americano. A partir de entonces la isla caribeña estuvo expuesta a las más intensas transformaciones en todos los aspectos de la vida social.

En la etapa histórica que abarca de 1959 a 1965, periodo en el que se construyeron los pilares del sistema socialista cubano, la Revolución vivió momentos importantes para su desarrollo. La cultura como referente primordial en la conformación de una nueva modernidad histórica, desde el comienzo fue centro de innovaciones que respondían al interés del recién creado estado. Las primeras iniciativas de la época demostraron la disposición política dirigida a establecer un 
campo cultural que diera paso a la expresión de todo tipo de creación artística y que legitimara la fundación de un saber revolucionario.

Las revoluciones presuponen un proceso de subversión de la totalidad social, en el que la cultura desempeña un papel fundamental en el establecimiento y legitimación del nuevo orden. El cambio político debe estar acompañado de la transformación de las bases culturales de la sociedad a fin de incorporar en la mentalidad colectiva la transformación efectuada. Convertir el proceso en un acto de innovación en todos los aspectos, que elimine las viejas costumbres, destruya el ancien régimen y edifique nuevos patrones intelectuales.

En el caso cubano, la cultura estuvo desde el primer momento en la agenda del Gobierno Revolucionario como un objetivo esencial para lograr a plenitud el cambio que se deseaba. Adquirió así un carácter de comprometimiento con el momento histórico. "(...) La política (sobre todo la revolucionaria) no es algo externo a la cultura (...). Es parte de la misma cultura" (Kohan, 2006, p. 8). Entre sus principales funciones se encontraban la de estabilizar los profundos desequilibrios, impulsar las fuerzas poderosas de la nacionalidad cubana y abrir caminos para la expresión creadora.

El campo cultural, en la etapa fundacional de 1959 a 1965, constituyó un espacio social en el cual convergieron la acción estatal y la creación artística agudizada por las nuevas oportunidades brindadas por el estado. En 1959 se creaba el Instituto Cubano del Arte e Industria Cinematográficos (ICAIC) bajo la dirección de Alfredo Guevara. El 28 de abril de 1959, mediante la Ley № 229, fue fundada la Casa de las Américas, con la dirección de Haydée Santamaría Cuadrado, heroína del Moncada. El acto de inauguración, el 4 de julio, fue presidido por el doctor Armando Hart, ministro de Educación. La institución deseaba ser la representación cultural del estado cubano en América Latina y para lograrlo proyectó un discurso que respondía al nuevo momento. La Casa fue establecida en función de representar los intereses del propósito revolucionario tanto al interior como al exterior, resultó ser un mecanismo de legitimación del nuevo poder estatal. 
El papel desempeñado por Casa de las Américas en el esquema cultural revolucionario de 1959 a 1965 se puede apreciar en los números esenciales de la revista Casa de las Américas $^{3}$. Durante este primer periodo, la publicación resultó ser la voz de la institución. Asimismo las premiaciones efectuadas en el Concurso Literario $^{4}$, de mismo nombre que la entidad, es otro referente importante para comprender las intenciones culturales de Casa.

El 12 de junio de 1959 se creaba el Teatro Nacional, su primera directora fue Isabel Monal Rodríguez. Con la fundación de la Imprenta Nacional, la edición y tirada masiva de libros fue recurrente en estos años. En 1961 se constituía el Consejo Nacional de Cultura, encargado de una política de desarrollo para esta área, y en 1962 se erigía la Escuela Nacional de Arte, comenzando desde entonces un amplio proceso de democratización de la cultura.

La apertura editorial en el sector de la prensa cultural jugó un papel fundamental en la expresión de los intelectuales cubanos. Entre los años 1959 a 1962 saldrían a la luz pública importantes revistas que marcaron pautas y posiciones: Lunes de Revolución en marzo de 1959, Cine Cubano en junio de 1960, la revista Casa de las Américas en julio de 1960 y La Gaceta de Cuba en abril de 1962. La esfera intelectual reescribía a diario sus formas y su funcionamiento, aparecían nuevos agentes, instituciones, medios de expresión y temáticas.

La iniciada fase de búsqueda y experimentación abría camino a una etapa de explosión creadora que predominó durante toda la década del 60 . Como parte de la misma, institucionalizar fue una premisa del estado que hizo eco en la vida artística e intelectual. "La Revolución Cubana fue creando su institucionalidad tanto política como civil- a partir de la asunción misma de la victoria como un hecho legítimo y legitimador a la vez" (Alonso, 2002, p.36). Las instituciones que se creaban sobre la marcha respondían a cambios radicales en todos los niveles sociales. Se erigían como mediadoras en la construcción de una hegemonía

\footnotetext{
${ }^{3}$ En este trabajo se utilizara cursiva para referirse a la revista Casa de las Américas.

${ }^{4}$ Originalmente el nombre del concurso era Concurso Literario Hispanoamericano, en 1964 pasó a llamarse Concurso Literario Latinoamericano y finalmente en 1965 adquiere su título actual: Premio Literario Casa de las Américas.
} 
enmarcada en una nueva reorientación política y además jugaban un papel cardinal en la articulación de un pensamiento ideológico. La variable cultura dentro del proceso de legitimación del poder revolucionario resultaba fundamental para articular una relación entre los creadores y el estado.

El nacimiento de una política de este corte estuvo marcado por un contexto de definiciones, precedido por la declaración del carácter socialista de la Revolución en marzo de 1961. A partir de la censura del documental PM, realizado por Orlando Jiménez Leal y Sabá Cabrera Infante, había salido a relucir una fuerte polémica en torno a la libertad creadora del artista y su posición respecto al compromiso de su obra. El discurso del Comandante en Jefe Fidel Castro Ruz, en las reuniones efectuadas con los intelectuales en la Biblioteca Nacional en el mes de junio, conocido como Palabras a los Intelectuales y el I Congreso de Escritores y Artistas de Cuba en agosto fueron los principales acontecimientos que establecieron la posición del estado en este sentido.

\section{La Casa de las Américas, una institución de la Revolución}

En la reestructuración de la sociedad civil $^{5}$ cubana resultaba esencial instituciones de tipo cultural que dotaran al campo intelectual de centros de reflexión y generadoras de un pensamiento y posturas ideológicas que legitimaran el proceso que se vivía. Para conocer el desarrollo cultural de una época o una etapa determinada de un país nada mejor que una entidad productora de un pensamiento intelectual como Casa de las Américas. Esta institución se encontraba inmersa en un convulso presente nacional, estimulada por el carácter mutable y polémico del periodo.

En función de entender el discurso de Casa de las Américas como parte de un todo revolucionario y como resultante de una intención estatal, el acercamiento a la producción editorial de la institución mediante su revista y su principal acción cultural (Concurso literario anual) resulta fundamental. Los años de 1959 a 1965 son esenciales para profundizar en dichas cuestiones.

\footnotetext{
5 "De esta manera se plantea que la sociedad civil la conforman un conjunto de organismos, que posibilitan la dirección intelectual y moral de la sociedad. Así, se encuentra articulada por múltiples organizaciones de carácter cultural, educativo, religioso mediante las cuales se difunden la ideología, los intereses y los valores de la clase que domina al estado" (Acanda, 2002, p.248).
} 
Casa propuso materiales, formas y poéticas que reafirmaron su condición de proyecto revolucionario. Creada gracias a una iniciativa estatal y no por gestiones autónomas del campo intelectual, responde a una intención política de generar desde la cultura un pensamiento revolucionario. El propio diseño de la dirección a cargo de Haydée Santamaría como rectora principal, vinculada a figuras claves dentro del gobierno revolucionario, evidencia una intención política que marcó el desarrollo de la propia institución.

Para establecer vínculos con América Latina se fomentó un programa que difundía dentro de la creación artística aquello que encarnara lo revolucionario y todas sus posibles variantes estéticas y discursivas. Se perseguía ante todo establecer un lazo entre el naciente estado y los pueblos del continente a través de su identidad y sus manifestaciones. Un espacio que permitiera abolir el pensamiento colonizador y colonizado para crear un pensamiento marcado por la identidad y lo propio. Bien se rememoraba un decenio después en las páginas de la revista:

Casa nacida precisamente con la revolución, para cumplir la tarea que ella nos encomendara, avivar, en el orden cultural, los lazos que deben unir entre sí a los pueblos de nuestra América, reconocerse unos a otros como integrantes de una comunidad supranacional cuya entrada en la historia mayor ha comenzado con esta revolución (...). (“Diez”, 1970, p.215)

La Casa se presentaba como un centro totalmente novedoso y sin precedentes en Cuba que permitía el constante intercambio y acercamiento a los intelectuales latinoamericanos.

La institución defendió como máxima al intelectual comprometido con la liberación y con las luchas revolucionarias latinoamericanas. Trabajaba además para proyectar sobre el continente la idea que se deseaba ofrecer de la Revolución, por consiguiente fue una vía de exportación directa de la misma.

Decía Ambrosio Fornet en Apuntes para el plano de una Casa:

La Casa era la puerta que daba a la revolución, que a su vez no era solo este ámbito político y humano enmarcado en la isla sino la perspectiva desde la que podía contemplar, como un paisaje fabuloso, el futuro previsible de nuestra América. (Quintero, 2002, p. 173) 
En alguna medida la Revolución, a través de la institución, pudo expedir la nueva cultura modélica para el continente. "Se pretendía influir más allá de los estrechos límites de nuestra Isla. América Latina era una pradera seca y Cuba, se creyó que sería la chispa que la incendiaría" (Otero, 1997, p.13).

Casa de las Américas, como su nombre indica, presenta una declaración de objetivos específicos: trabajar sobre la identidad, crear una zona de confluencia para todo el continente, tender un puente para comunicar los balcanizados países latinoamericanos que se presentaban tan aislados entre sí, así como aspirar a lograr discursos propiamente autóctonos: “(...) desde su título, no solo semantizó una zona arquitectónica diseñada para un trabajo cultural, sino que además impulsó un proyecto unificador de las identidades del continente" (Quintero, 2002, p.102). Empresa que también persiguió la revista y el Premio. Casa se establecía como un lugar multidisciplinario que fomentaba los espacios internacionales, un centro para la concordancia creadora mediante una política múltiple, que además viabilizara la posición democratizadora que impulsaba la Revolución. Su carácter no quedó limitado a lo netamente latinoamericano, sino a una mezcla de discursos en torno a lo latinoamericanista y revolucionario.

Los objetivos principales perseguían dar a conocer las expresiones propias del continente, estimular la creación literaria y artística entre los escritores, teatristas, plásticos y músicos, así como la tarea de investigadores latinoamericanos y caribeños, la difusión de obras, la investigación y la organización de encuentros entre intelectuales. La proyección institucional de Casa de las Américas y sus líneas de trabajo, incluían no solo las publicaciones que caracterizaron su quehacer sino también actividades que reflejaran su propio discurso: conferencias de Ezequiel Martínez Estrada, Subdesarrollo y Revolución de Enrique Gonzales Pedrero; diálogos con Miguel Ángel Asturias; lecturas de Pablo Neruda, José Rodríguez Feo y mesas redondas sobre La Revolución Cubana y El escritor americano ante la Revolución; son algunos de los ejemplos.

En este contexto es imposible obviar, como ya se ha mencionado, la aparición y la influencia inmediata de Palabra a los intelectuales. El discurso tuvo onda repercusión, tanto en la isla, como para toda el área geográfica al marcar 
decisivamente la voz pública de las culturas de izquierda latinoamericana en los años 1960. Entraba en debate cual podría ser el rol del intelectual en una situación cambiante, y hasta qué punto es o no permisible un diálogo entre el nuevo poder y el campo intelectual.

La primacía de la Revolución frente a cualquier situación era patrón formulado y por tanto:

(...) Su derecho a controlar la actividad intelectual y la libertad de expresión en todo lo que resultara necesario, reclamar a los intelectuales tener fe o confianza en la Revolución, respaldar al Consejo Nacional de Cultura sin dejar a su pleno arbitrio el campo cultural y fortalecer la política de institucionalización estatal y de organizaciones sociales. (Martínez, 2011, p.1)

Palabra a los intelectuales, fue la posición del gobierno frente a los problemas del arte y la literatura, sin duda constituyó la formulación revolucionaria de una política al respecto. El texto produjo una imagen de la institucionalidad que regiría la actividad del intelectual cubano; ser revolucionario era creer en las nuevas instituciones, en el nuevo interior. Palabra... funda una tipografía moral e institucional para las operaciones del campo intelectual. Para Casa, las líneas a seguir de este plano de la política se convirtieron en jurisdicción. Nicolás Guillén lo apuntaba en el Informe de 1961 al 1er Congreso de Escritores y Artistas de Cuba: "Digamos (...) que los intelectuales no forman una clase social. Bajo el socialismo el intelectual sirve al pueblo (...)" (Guillén, 1961, p.12), se trataba de hacer un arte que respondiera a la realidad revolucionaria y su formación histórica.

\section{Discurso y acción intelectual: Casa de las Américas y el Premio Literario}

Casa de las Américas es emitida por primera vez el 6 de julio de 1960 bajo la dirección de Haydée Santamaría y la responsabilidad de Fausto Masó y Antón Arrufat. La revista se insertó en una etapa marcada por el auge editorial. Con Lunes de Revolución ${ }^{6}$ compartió diversos puntos en común, además de las

\footnotetext{
${ }^{6}$ Lunes de Revolución semanario cultural surgido en marzo de 1959, dirigido por Guillermo Cabrera Infante y Pablo Armando Fernández, circuló por todo el país hasta noviembre de 1961. Escritores como Antón Arrufat, Calvert Casey, José A Baragaño, Edmundo Desnoes, Heberto Padilla, Rine Leal, Virgilio Piñera y muchos más, contribuyeron con su pluma al desempeño de la publicación. En sus artículos y ensayos se expresaron creadores de diferentes ideologías y generaciones. Tampoco fue exclusivamente literario, en él se publicaba lo mismo cuestiones de
} 
semejanzas en la elección de temáticas los fundadores de la revista fueron estrechos colaboradores del semanario cultural. Estas dos publicaciones, Lunes y Casa, fueron las primeras que se supieron más leídas en el campo intelectual cubano, rebasando los patrones de consumo cultural de antes del triunfo revolucionario.

En el número uno de Casa de las Américas, se declaraba, en una editorial titulada "como haremos", los propósitos que perseguía la publicación:

Esta revista cree, tal vez ingenuamente, en la existencia de una concepción de la vida hispanoamericana. Esta revista es una esperanza, incierta y riesgosa de la posibilidad de cambiar la realidad. Porque, si existe América no es la que encontramos cada día, deshecha y superficial, sino la que en política ha demostrados que la utopía puede hacerse real, y que por tanto la Revolución no es una falacia. Es una razón ante la cual pomos aceptar morir sin dramatismo pero conscientemente. Y la literatura es también esa aventura de transformar la realidad americana. (1960, p.3)

La revista se presentaba como voz de la institución en su intención de emanar hacia las comunidades latinoamericanas las ideas revolucionarias. En la contraportada de cada número hasta 1965 recalcaba: "La Casa de las Américas es una institución cultural dirigida a servir a todos los pueblos del continente en su lucha por la liberación." Los artículos referentes a las condiciones sociales y políticas de América Latina ocupaban un importante espacio de la publicación que deseaba darle seguimiento a la realidad latinoamericana. En editorial titulada San José y la traición, referente a los acontecimientos de la OEA se planteaba: "Un día la Cordillera de los Andes puede ser otra Sierra Maestra y la Reforma Agraria convertirse en algo eficaz (...)" ("Editorial”, 1960, p. 5).

Casa de las Américas aparece en un contexto latinoamericano en el que grandes escritores del continente militaban en organizaciones de izquierda o se perfilaban a favor de procesos políticos transformadores. Este pensamiento revolucionario cobraba fuerza en parte, estimulado por el propio triunfo de 1959 y la institución y su revista se convirtieron en un medio oportuno para establecer confluencias entre ellos y la Revolución Cubana.

actualidad política, cultural o artísticas. Desde sus páginas se pensaba la realidad cubana con una intención modernizadora. 
Continuamente, Casa de las Américas exponía la cuestión de qué tipo de intelectual demandaba la realidad histórica continental. Un intelectual de izquierda, conectado con un proceso revolucionario, no solo el cubano sino al que se aspira en toda América. Las declaraciones explicitas sobre el compromiso intelectual constaban en casi todos los números de la revista. Desde el primero, Virgilio Piñera en "Un testimonio sobre el 1ero de mayo" (1960, p.32), recalcaba ,sin aparente intención, los escritores que habían dicho presentes en fecha tan distintiva estableciendo una brecha entre aquellos que manifestando su comprometimiento se vinculaban a las actividades sociales y políticas y los otros que se mantenían al margen. La idea del compromiso fue una de las temáticas que más auge tuvo en las páginas de la publicación como centro de discusión y como fundamento ideológico que debía guiar las actitudes del creador.

A tono con esta línea de pensamiento para 1961 Paul Baran, con un fuerte acento gramsciano recalcaba:

El intelectual es así en esencia un crítico social, una persona cuya preocupación es identificar, analizar, y de esta manera ayudar a vencer los obstáculos que impiden la realización de un orden social más humano y racional. Como tal él se convierte en la conciencia de la sociedad y el vocero de esas fuerzas progresistas que ella contiene en cualquier periodo de la historia. (p.13)

En contrapartida el poeta salvadoreño Roque Dalton en el ensayo "Poesía y militancia en América Latina" ofrecía otras observaciones en torno al tema del creador y su posición frente a cierta realidad:

El poeta debe ser fundamental fiel con la poesía, con la belleza. Dentro del caudal de lo bello debe sumergir el contenido que su actitud ante la vida y los hombres le imponga como gran responsabilidad de convivencia. Y aquí no caben los subterfugios ni la inversión de los términos. El poeta es tal porque hace poesía, es decir porque crea una obra bella. (...) Hay que desterrar esa concepción falsa, mecánica y dañina según la cual el poeta comprometido con su pueblo y con su tiempo es un individuo iracundo o excesivamente dolido que se pasa la vida diciendo, sin más ni más, que la burguesía es asquerosa, que lo más bello del mundo es una asamblea sindical y que el socialismo es un jardín de rosas dóciles bajo un sol especialmente tierno. (1963, p.15)

La responsabilidad intelectual defendía una condición que significaba la verdadera militancia manifestada en la esfera artística y literaria, una producción intelectual cercana a los fenómenos políticos, sociales y económicos. Una toma de 
conciencia respecto a la función del creador para desempeñar un papel activo en la vida de la sociedad. "Si este tiempo latinoamericano se nos presenta urgido, desgarrado, contradictorio, como en época de revolución inminente, así también se nos presentará el panorama de la creación artística" (Rama, 1964, p.3).

La revista, en su interés por exaltar estas cuestiones, en entrevista a escritores cubanos preguntaba como centro de atención: ¿Cuál es la función del escritor en la revolución y el significado que esta tenía en la obra? ("Entrevistas", 1964, p.139) De esta forma, emitía y, comunicaba al continente un mensaje que refrendaba el ideal de intelectual comprometido. Hacía posible que las voces realmente identificadas se escucharan, publicaran y dejaran eco.

Para el año 1967, en número dedicado a la temática del intelectual comprometido, se retomaba el asunto a través de las voces de Julio Cortázar, Vargas Llosa, René Depestre, entre otros. En "Situación del intelectual en América Latina" Mario Benedetti (1967) señalaba que el escritor tiene doble responsabilidad: la de su arte y la de su contorno. Citaba a Arthur Miller cuando refería que "el hombre está dentro de la sociedad y la sociedad dentro del hombre" (...) "No creo en el compromiso forzado, ni en la militancia que desvitaliza un tema, pero tampoco creo en la línea divisoria entre obra literaria y responsabilidad humana del escritor" (1967, p. 35). Desde la revista en voz de los intelectuales de avanzada, se retomaba constantemente esa necesidad de un arte nuevo, de una política nueva y de cambio.

Constantemente en la publicación se exaltaban los méritos del proceso cubano no solo mediante el ensayo o el artículo periodístico, sino también a través de la poesía: "Revolución, eres una / paloma que va volando.../ ¡Ay, paloma que nació/ sobre la Sierra y bajó al llano, / en la sierra y llano creció!/ Muchacha recién nacida, / dame la mano, / toma mi vida (...)" (Guillén, 1960b, p. 7).

El semanario Lunes de Revolución, con motivo de saludar al concurso Casa de las Américas realizó una serie de entrevistas, entre ellas, al escritor mexicano Fernando Benítez quien comentaba que "Cuba es hoy la más firme incitación revolucionaria de América Latina. Aquí se está realizando la obra que toda América Latina debería imitar" (“Un jurado”, 1960, p. 2). 
Julio Cortázar (1970) en "Algunos aspectos del cuento" con razón alegaba que:

Aquí, más que en ninguna otra parte, se requiere hoy una fusión total de dos fuerzas, la del hombre comprometido con su realidad nacional y mundial y la del escritor lúcidamente seguro de su oficio... sino su obra no va más allá del mero ejercicio estético(...). (p.184)

Queda advertido un discurso que traspasaba los límites latinoamericanistas puramente, era un llamado a la arena pública, a una transformación de juicios, de ideas. Se buscaba erigir un hombre nuevo, esteta, pero que construyera su discurso artístico en función de un proceso, que no se aislara de su realidad. Estas ideas encontraron acogida y seguimiento en figuras como las que hemos abordado y en otras propiamente americanas: Manuel Galich, José Pedroni, Ezequiel Martínez. Casa de las Américas quería repensar la Patria grande, a la luz de la verdad revolucionaria.

A medida que la Revolución se radicalizaba y definía, las temáticas del campo intelectual iban tomando un mayor cauce político. El compromiso y la función del escritor o artista se vinculaban cada vez más a lo social. Estas condicionantes impusieron cambios y discusiones en la vida de muchas de las instituciones y publicaciones de la época. En Casa de las Américas, a partir de 1965, se deciden operar transformaciones en apariencia sencillas, pero que respondían a giros políticos e ideológicos. Se renombra el "Consejo de redacción" como "Comité de colaboración," un título supuestamente más pertinente a las necesidades de la revista. La rectoría de la revista pasa de Haydée Santamaría a Fernández Retamar y es suspendido el Consejo de Redacción guiado por Antón Arrufat tras conflictos internos ${ }^{7}$.

\footnotetext{
${ }^{7}$ Desde el primer número hasta el treinta Haydee Santamaría fue la directora de la publicación. Sin embargo las variaciones en torno a la redacción de la revista se registran continuamente: en el número 1 se recogen como Responsables de la Revista a Antón Arrufat y Fausto Masó, en el número 6 se transforma en Consejo de Redacción que incluía a A. Arrufat, Ezequiel Martínez Estrada y Elvio Romero. En el número 8 el Secretario de Redacción fue Pablo Armando Fernández. El número 20-21 tenía como Consejo de Redacción a Haydee Santamaría, Ezequiel Martínez Estrada, Manuel Galich, Julio Cortázar y Enmanuel Carballo y como Jefe de Redacción a Antón Arrufat. Para el numero 30 aparece como Director Roberto Fernández Retamar y se sustituye la idea de un Consejo o Jefes de Redacción por un Comité de Colaboradores de carácter amplio.
} 
Arrufat indicaba que a la revista se le empezaban a ver "cosas malas" (Lie, 1996, p.28-30). Por ejemplo, el hecho de publicar alguna personalidad que no tuviera que ver directamente con la revolución o que no estuviera "definida políticamente" era tema de contradicción. En este período la mayoría de las figuras literarias de izquierda tanto de Cuba como de América son las que publicaban reiteradamente, además muchas eran premiadas o formaban parte del jurado en el concurso que convocaba la institución.

Existía un hecho determinante que también influyó en los cambios de 1965. Los redactores y gran parte de los colaboradores cubanos de la revista desde 1960 hasta 1965 habían estado estrechamente vinculados al magazine Lunes de Revolución, el cual había tenido un cierre polémico por lo que desde entonces cualquier lazo con el semanario era visto a través de un prisma políticamente incorrecto. En aquella circunstancia de contradicciones "Haydée propone que se haga una revista de carácter más político, menos literario (...) una revista más institucional, que refleje también el trabajo de la Revolución en la cultura (...)" (Lie, 1996, p. 276).

Ante tales disyuntivas, Arrufat renuncia al puesto dentro de la revista y Fernández Retamar asume la dirección. A partir del número 30 se establecen estrategias de diferenciación respecto a la orientación anterior tales como el detrimento del contenido literario a favor uno político. ${ }^{8}$ Con la nueva dirección y el recién creado Comité de Colaboración se imprime una mayor solidez en las funciones que dan fin a la inestabilidad de colaboradores en la redacción de la revista.

Aunque esa ruptura no se reconozca como una de los momentos polémicos de la década del 60, indudablemente el cambio en la directiva y las nuevas líneas en la redacción a partir de 1965, forman parte de un complejo campo cultural en el que a diario se vivían debates y contradicciones que respondían a posiciones

\footnotetext{
${ }^{8}$ Revisando los índices de la revista a partir de 1965 se aprecia un fuerte impulso de artículos que indagan en las condiciones revolucionarias de latinoamericana. Algunos ejemplo: de Regis Debray: América Latina: Algunos problemas de estrategia revolucionaria, Manuel Maldonado Puerto Rico: libertad y poder en el Caribe, Augusto Roa Bastos Paraguay ante la necesidad de su segunda independencia. Además ante la agresión de los Estados Unido a República Dominicana en 1965 Casa... emitía una Declaración en la que denunciaba la actitud norteamericana y exigía el retiro de las tropas mercenarias.
} 
ideológicas y políticas y que en última instancia buscaban edificar un espacio intelectual que respondiera al contexto histórico.

Como parte del amplio proceso de institucionalización y de las ideas jóvenes y creadoras de Casa, aparece el Concurso Literario Hispanoamericano citando a la América Latina en su totalidad y otorgado según las convocatorias a las diferentes categorías literarias: cuento, novela, teatro, ensayo, poesía. E premio fue una de las principales acciones culturales de la institución, de amplia referencia en sus primeros años. Los jurados para los diferentes géneros, se conformaron con personalidades de disímiles países de Latinoamérica. Se iniciaba así una relación con un grupo de intelectuales hasta el momento no vinculados con la realidad cubana. Muchos de los participantes, desconocidos hasta entonces, salieron a la palestra pública gracias al llamado de Casa. A través del lauro se manifestaban acciones culturales tanto de producción como distribución.

En la primera edición en 1960 resultaron condecorados José Soler Puig, por su novela Bertillon 166; Jorge Enrique Adoum, por su poemario Dios trajo la sombra; Ezequiel Martínez Estrada, por el ensayo Análisis funcional de la cultura; Cuentos de vida y muerte, de José María López Valdizón y Santa Juana de América, de Andrés Lizarraga, fueron premiados en cuento $y$ teatro respectivamente. Sin duda alguna, los ganadores del premio debían trabajar problemáticas a tono con las exigencias de un jurado que demandaría doblemente cualidades estéticas y textos que tributaran a las realidades cubana $y$ latinoamericana. Lo anterior respondía al mismo perfil discursivo que perseguía la institución.

En una entrevista al novelista y ensayista ecuatoriano, Benjamín Carrión (1960), quién fue parte del jurado en la especialidad de poesía, argumentaba que podía resultar difícil discernir cuál era la mejor poesía en libros de igual calidad. En este caso, acudiría al más racional, a la menos oscura y concluye que el concurso era una idea excelente y sus resultados favorecerían a la Revolución. Nicolás Guillén, que también integraba dicha comisión, expresó sus motivos a la hora de aceptar participar en el evento: no podía negarle el apoyo a Haydée y a los momentos de reconstrucción espiritual que vivía la Patria. El poeta nacional era 
del criterio que la obra premiada debía estar en concordancia con la instancia revolucionaria, lo ideal era seleccionar un libro que expresara mediante su poética los profundos cambios que acontecían en el universo latinoamericano. (Guillén, 1960a, p. 8)

El premio de poesía otorgado a Jorge Enrique Adoum provocó insatisfacciones en algunos miembros del jurado. Según afirmaba Virgilio Piñera el resultado era consecuencia de las características propias de la obra de Nicolás Guillen, poeta que reunía lo revolucionario y lo artístico. En este caso, Dios trajo la sombra correspondía a esta concepción. Piñera (1960) abogaba por seleccionar como galardonado a Poesía, revolución del ser de José A Baragaño "por el hecho mismo de postular al hombre que vive en nuestro tiempo, por sacar a libre plática el alma de este hombre, resulta de mayor utilidad y eficacia poética" (p. 9). El dramaturgo cubano reconoce el avance y la importancia de la poesía social pero acotaba que "el que no participara en esta cruzada era tildado de oveja negra" (Piñera, 1960, p. 9). Igualmente, estimó que premiar a Adoum significaba premiar la antipoesía pues no se postulaba el hecho poético desde la poesía en si misma sino desde lo económico y político.

En estos primero años resultaron premiados títulos bastantes sugerentes: en 1961 se seleccionaron de Dora Alonso, Tierra inerme en novela y de Luis E. Valencian, Realidad y perspectiva de la Revolución cubana en ensayo. Como parte del jurado se encontraban Ezequiel Martínez Estrada, Ramiro Guerra y Carlos Rafael Rodríguez por ensayo y por novela José Bianco y José Rodríguez Feo. Para el año 1962 los ganadores del concurso fueron Daura Olema con Maestra voluntaria por novela, Raúl González de Cascorro con Gente de Playa Girón en cuento y Gumersindo Martínez Amengual: Presencia de la reforma agraria en América en ensayo y Fayad Jamís con Por esta libertad en poesía.

El poemario ganador representaba un canto social, que legitimaba un momento histórico: "Por esta libertad de canción bajo la lluvia / habrá que darlo todo / por esta libertad de estar estrechamente atados / a la firme y dulce entraña del pueblo / habrá que darlo todo..." (Jamís, 1962, p. 31). La isla vivía un período complejo, en el que el conflicto con los Estados Unidos era bastante tenso. Cuba 
era un país declarado socialista y por lo tanto premiar un poema que respondiera al momento histórico, que lo reflejara era lo más válido por lo que resultaba muy oportuno condecorar el canto de Fayad Jamís.

El Concurso literario de Casa de las Américas buscaba ameritar la obra militante, que estuviese en comunión con realidades, que validara el discurso que nace y divulgara hacia las comunidades de la región el pensamiento revolucionario. Bien decía Arrufat (1959):

(...) que revolución implica la creación de nuevos valores, ésta nueva concepción revolucionaria puede enjuiciar el valor permanente de la obra de nuestros escritores; "la revolución no solo ha repartido tierras sino que ha creado un nuevo o diferente concepto de valor (...) ya no es posible (literariamente hablando) la concepción de temas que no comprometen ningún valor. (p.16)

La presencia de los jurados extranjeros en Cuba aseguraba la divulgación de la realidad cubana: "nosotros casi todos o todos, hacemos periodismo y a nuestro regreso diremos la verdad de la revolución cubana y sus propósitos" (1960, p.3), decía el ecuatoriano Carrión a Lunes de Revolución. Un constante crear de vías de emisión, de divulgación de qué es la revolución se aseguraba. Casa de las Américas, a través de su revista, de su premio, de los jurados de estos, de los intercambios funcionaba como una senda excelente de canalización de un proyecto a realizar en el continente. En el citado número 44 de Lunes se entrevistó a Fernando Benítez escritor mexicano, y se le hizo la siguiente pregunta: ¿Cree usted que nuestra revolución ejerce influencia sobre el resto del continente? A lo que el escritor respondía: "es una influencia evidente, Cuba como la más firme incitación revolucionaria de América Latina, donde se ha realizado una obra que América Latina debería imitar" (Carrión, 1960, p.2).

\section{A modo de Conclusión}

Durante sus primeros años de existencia Casa de las Américas, ejerció una importante labor cultural e intelectual. Si pensamos en el ambiente que nace la Revolución, y la situación de la América en general vemos que fue más que pertinente la aparición de una institución de este tipo:

(...) porque desde los primeros momentos de nuestra Revolución, nosotros sentimos el valor de la cultura latinoamericana, la cultura de nuestros pueblos. Y para nosotros la verdadera cultura de Nuestra América tenía tanta importancia 
como cualquiera de los trabajos más importantes que pudiera hacer una Revolución. (Santamaría, 1987, p. 71).

Casos como la expulsión de Cuba de la Organización de Estados Americanos en 1962, sirvieron para distanciar políticamente al gobierno revolucionario de los estados latinoamericanos. Como contrapartida la Casa se convirtió en un punto de aproximación con los pueblos del sur.

Decía Jorge Adoum (Casa de las Américas, 1999):

Gracias a esta institución cultural la revolución hacía hincapié en el ser humano no solo como creador de una nueva realidad social sino también como un ser dotado de capacidad para reflejar esa realidad, contribuir a cambiar e incluso recrearla 0 suplantarla(...).(p. 26)

Casa de las Américas, quería una mirada latinoamericana más profunda y analítica. La aspiración tenía como punto de partida un país que había subvertido las bases de un sistema y creaba sobre la marcha un estado revolucionario. La promoción de instituciones culturales tributaba no solo a la idea de progreso sino también al cambio de una mentalidad colectiva. La Casa arranca con la esperanza de influir, desde un espacio, una publicación, un premio, desde un país, para obtener un cambio generalizado. Lograr para los países de la América su propia revolución, política, socioeconómica y cultural en semejanza a la cubana.

\section{Referencias Bibliográficas}

Acanda, J.L. (2002). Sociedad Civil y Hegemonía. La Habana: Centro de Investigación y Desarrollo de la Cultura Cubana Juan Marinello.

Alonso, A. (2002). La institucionalidad civil y el debate sobre la legitimidad. Temas, 29, 36-45.

Arrufat, A. (1959). Idea de la Revolución. Lunes de Revolución, 35,16.

Baran, P. (1961). El compromiso intelectual. Casa de las Américas, II (7),13.

Benedetti, M. (1967). Situación del escritor en América Latina. Casa de las Américas, VIII (45), 35.

Carrión, B. (1960). Un jurado internacional de escritores. Lunes de Revolución, (44), 3.

Cortázar, J. (1970). Algunos aspectos del cuento.Casa de las Américas. XI (63), 184. 
Casa de las Américas. (1999). Cultura y revolución. A 40 años de 1959. La Habana: Fondo Editorial Casa de las Américas.

¿Cómo haremos? (1960). Casa de las Américas, 1 (1), 3.

Dalton, R. (1963). Poesía y militancia en América Latina. Casa de las Américas, III (21), 15 .

Diez años de la revista Casa de las Américas. (1970).Casa de las Américas, XI (63), 215.

Editorial. (1960). Casa de las Américas, I (2), 5.

Entrevistas. (1964) Casa de las Américas, 4 (22-23),139.

Guillén, N. (1960a). Lunes de Revolución, 47,8.

Guillén, N. (1960b). Muchacha. Casa de las Américas, I (2), 7.

Guillén, N. (1961).Informe al I Congreso de Escritores y artistas cubanos. Casa de las Américas, II (8), 12.

Kohan, N. (2006). Pensamiento Crítico y el debate por las Ciencias Sociales en el seno de la revolución cubana. Recuperado de http://www.criterios.es/pdf/kohanpensamiento critico.pdf

Lie, N. (1996). Transición y transacción. La revista cubana Casa de las Américas 1960-1976. USA: Ediciones Hispamérica.

Martínez, F. (2011). La imprescindible tarea de recuperar la memoria. La Jiribilla, X (530). Recuperado de http://www.lajiribilla.cu/2011/n53007/53013.html

Otero, L. (1997). Llover sobre mojado. Una reflexión personal sobre la historia. La Habana: Editorial Letras Cubanas.

Quintero, J.C. (2002). Fulguración del espacio. Letras e imaginario institucional de la Revolución Cubana (1960-1971). Rosario: Beatriz Viterbo Editora.

Santamaría, H. (1987). Todo arte verdadero es política. En N. Nuiry (Ed.), Pensamiento y políticas culturales cubanas (Tomo II) (pp.66-71) La Habana: Editorial Pueblo y Educación.

Piñera, V. (1960).Un testimonio sobre el 1ero de mayo. Casa de las Américas, I (1), 32.

Piñera, V. (1960). Votos y Vates.Lunes de Revolución, 47, 9. 
Rama, Á. (1964). Diez problemas para el novelista latinoamericano. Casa de las Américas, IV (26), 3.

Un jurado internacional de escritores. (1960). Lunes de Revolución, 44, 2. 